\title{
GAUSSIAN MEASURE OF LARGE BALLS IN A HILBERT SPACE ${ }^{1}$
}

\section{CHII-RUEY HWANG}

\begin{abstract}
Let $\boldsymbol{P}$ be a zero mean Gaussian measure in a Hilbert space. The
\end{abstract} asymptotic behavior of $P\left\{\|x-b\|^{2}>\varepsilon\right\}$ as $\varepsilon \rightarrow \infty$ is studied in this note.

Let $P$ be a Gaussian measure in a separable Hilbert space $\mathcal{H}$ and $b$ be an arbitrary fixed element in $\mathcal{H}$. We shall study how fast $P\left\{\|x-b\|^{2}>\varepsilon\right\} \rightarrow 0$ as $\varepsilon \rightarrow \infty$.

Without loss of generality, $P$ is assumed to be of mean 0 . Let $B$ be the covariance operator of $P$, and let the eigenvalues (corresponding eigenvectors) of $B$ be ordered by $\lambda_{1} \geqslant \lambda_{2} \geqslant \cdots\left(\left\{e_{i}\right\}\right)$. Let $k$ be the multiplicity of the largest eigenvalue, $x_{i}=\left\langle x, e_{i}\right\rangle, b_{i}=\left\langle b, e_{i}\right\rangle$ and $a=\left(\sum_{1}^{k} b_{i}^{2}\right)^{1 / 2}$. Finally, let $F$ denote the distribution function of $\|x-b\|^{2}=\Sigma_{1}^{\infty}\left(x_{i}-b_{i}\right)^{2}$. The Laplace transform of $F$ is

$$
\begin{aligned}
\phi(c)= & \int_{0}^{\infty} c^{-c t} d F(t)=\prod_{i=1}^{\infty}\left[\left(1+2 c \lambda_{i}\right)^{-1 / 2} \exp \left(-b_{i}^{2} c\left(1+2 c \lambda_{i}\right)^{-1}\right)\right] \\
\psi(c)= & \int_{0}^{\infty} e^{-c t+t / 2 \lambda_{1}}(1-F(t)) d t \\
= & \left(1-\phi\left(c-\frac{1}{2 \lambda_{1}}\right)\right)\left(c-\frac{1}{2 \lambda_{1}}\right)^{-1} \\
= & \left(c-\frac{1}{2 \lambda_{1}}\right)^{-1}\left(2 c \lambda_{1}\right)^{-k / 2} \exp \left(\frac{a^{2}}{4 c \lambda_{1}^{2}}-\frac{a^{2}}{2 \lambda_{1}}\right) \\
& \times\left[\left(2 c \lambda_{1}\right)^{k / 2} \exp \left(\frac{a^{2}}{2 \lambda_{1}}-\frac{a^{2}}{4 c \lambda_{1}^{2}}\right)-\prod_{k+1}^{\infty}\left(1+2 c \lambda_{i}-\frac{\lambda_{i}}{\lambda_{1}}\right)^{-1 / 2}\right. \\
& \left.\quad \times \exp -\sum_{k+1}^{\infty} b_{i}^{2}\left(c-\frac{1}{2 \lambda_{1}}\right)\left(1+2 c \lambda_{i}-\frac{\lambda_{i}}{\lambda_{1}}\right)^{-1}\right] .
\end{aligned}
$$

If $a=0$, then

$$
\psi(c) \sim\left(2 \lambda_{1}\right)^{1-k / 2} \prod_{k+1}^{\infty}\left(1-\frac{\lambda_{i}}{\lambda_{1}}\right)^{-1 / 2} \exp \left(\frac{1}{2 \lambda_{1}} \sum_{k+1}^{\infty} b_{i}^{2}\left(1-\frac{\lambda_{i}}{\lambda_{1}}\right)\right)^{-1} c^{-k / 2}
$$

Received by the editors February 5, 1979.

AMS (MOS) subject classifications (1970). Primary 60B99; Secondary 60G15.

Key words and phrases. Asymptotic behavior, Gaussian measure, Hilbert space, Laplace transform, Tauberian theorem.

${ }^{1}$ This research was supported by NSF Grant MCS 76-80762. 
as $c \rightarrow 0$. By a Tauberian theorem [1], as $\varepsilon \rightarrow \infty$,

$$
\begin{aligned}
\int_{0}^{\varepsilon} e^{t / 2 \lambda_{1}}(1-F(t)) d t \sim & \frac{1}{\Gamma(k / 2+1)}\left(2 \lambda_{1}\right)^{1-k / 2} \prod_{k+1}^{\infty}\left(1-\frac{\lambda_{i}}{\lambda_{1}}\right)^{-1 / 2} \\
& \times \exp \left(\frac{1}{2 \lambda_{1}} \sum_{k+1}^{\infty} b_{i}^{2}\left(1-\frac{\lambda_{i}}{\lambda_{1}}\right)^{-1}\right) \varepsilon^{k / 2} .
\end{aligned}
$$

Since $e^{t / 2 \lambda_{1}}(1-F(t))$ is continuous and positive, L'Hôpital's rule is applicable. Hence we have

THEOREM 1. If $a=\left(\Sigma_{1}^{k} b_{i}^{2}\right)^{1 / 2}=0$, then

$$
P\left\{\|x-b\|^{2}>\varepsilon\right\} \sim K_{1} e^{-\varepsilon / 2 \lambda_{1}} \varepsilon^{k / 2-1},
$$

where

$$
K_{1}=\frac{1}{\Gamma(k / 2)}\left(2 \lambda_{1}\right)^{1-k / 2} \prod_{k+1}^{\infty}\left(1-\frac{\lambda_{i}}{\lambda_{1}}\right)^{-1 / 2} \exp \frac{1}{2 \lambda_{1}} \sum_{k+1}^{\infty} b_{i}^{2}\left(1-\frac{\lambda_{i}}{\lambda_{1}}\right)^{-1} .
$$

If $a>0$, then $\psi(c) \rightarrow \infty$ exponentially fast as $c \rightarrow 0$. The ordinary Tauberian theorem is not applicable here. We shall try another approach.

Let $\bar{b} \in \mathcal{H}$ such that $\overline{b_{i}}=0$ if $i \leqslant k$ and $\bar{b}_{i}=b_{i}$ otherwise. Then, for $\varepsilon$ large enough

$$
\begin{aligned}
& P\left\{\|x-b\|^{2}>\varepsilon\right\} \\
& \quad \leqslant P\left\{\|x-\bar{b}\|^{2}>(\sqrt{\varepsilon}-a)^{2}\right\} \sim K_{1}\left(\exp \frac{-1}{2 \lambda_{1}}(\sqrt{\varepsilon}-a)^{2}\right) \varepsilon^{k / 2-1} .
\end{aligned}
$$

On the other hand, $P\left\{\|x-b\|^{2}>\varepsilon\right\}$ may be regarded as $P_{b}\left\{\|x\|^{2}>\varepsilon\right\}$, where $P_{b}$ has mean $-b$ and covariance $B$. It is easily seen that $P_{b}$ is equivalent to $P_{\bar{b}}$ and

$$
\frac{d P_{b}}{d P_{5}}(x)=\exp \left(\frac{-\sum_{1}^{k} x_{i} b_{i}}{\lambda_{1}}-\frac{a^{2}}{2 \lambda_{1}}\right)
$$

Therefore

$$
\begin{aligned}
& P\left\{\|x-b\|^{2}>\varepsilon\right\}=P_{b}\left\{\|x\|^{2}>\varepsilon\right\} \\
& =\int_{\|x\|^{2}>\varepsilon} \exp \left(\frac{-\sum_{1}^{k} x_{i} b_{i}}{\lambda_{1}}-\frac{a^{2}}{2 \lambda_{1}}\right) d P_{\bar{b}}(x) \\
& >\left(2 \pi \lambda_{1}\right)^{-k / 2} \int_{x_{1}^{2}+\cdots+x_{k}^{2}>\varepsilon} \exp \left(\frac{-1}{2 \lambda_{1}} \sum_{i=1}^{k}\left(x_{i}+b_{i}\right)^{2}\right) d x_{1} \cdots d x_{k} \\
& =\left(2 \pi \lambda_{1}\right)^{-k / 2} \int_{x_{1}^{2}+\cdots+x_{k}^{2}>\varepsilon} \\
& \quad \quad \times \exp \left(\frac{-1}{2 \lambda_{1}}\left[\left(x_{1}-a\right)^{2}+x_{2}^{2}+\cdots+x_{k}^{2}\right]\right) d x_{1} \cdots d x_{k} .
\end{aligned}
$$


(To get the last equality, just rotate the vector $\left(-b_{1}, \ldots,-b_{k}\right)$ to the first coordinate.)

For $k>3$, change the last $k-1$ coordinates to polar coordinates; (3) becomes

$$
\begin{aligned}
& \left(2 \pi \lambda_{1}\right)^{-k / 2} \frac{2 \pi^{(k-1) / 2}}{\Gamma((k-1) / 2)} \int_{\substack{x_{1}^{2}+r^{2}>\varepsilon \\
r>0}}\left(\exp -\frac{\left(x_{1}-a\right)^{2}+r^{2}}{2 \lambda_{1}}\right) r^{k-2} d r d x_{1} \\
& =\left(2 \pi \lambda_{1}\right)^{-k / 2} \frac{2 \pi^{(k-1) / 2}}{\Gamma((k-1) / 2)} \\
& \quad \times \int_{0}^{\pi} \int_{\sqrt{\varepsilon}}^{\infty}\left(\exp \frac{\rho^{2}+a^{2}-2 \rho a \cos \theta}{2 \lambda_{1}}\right) \rho(\rho \sin \theta)^{k-2} d \rho d \theta .
\end{aligned}
$$

By L'Hôpital's rule

$$
\begin{aligned}
& \lim _{\varepsilon \rightarrow \infty} \frac{\int_{0}^{\pi} \int_{\sqrt{\varepsilon}}^{\infty}\left(\exp \left(\left(2 \rho a \cos \theta-\rho^{2}-a^{2}\right) / 2 \lambda_{1}\right)\right) \rho(\rho \sin \theta)^{k-2} d \rho d \theta}{\varepsilon^{(k-3) / 4} \exp \left(-(\sqrt{\varepsilon}-a)^{2} / 2 \lambda_{1}\right)} \\
& =\lim _{\varepsilon \rightarrow \infty} \frac{\varepsilon^{(k-2) / 2}\left(\exp -\left(\varepsilon+a^{2}\right) / 2 \lambda_{1}\right) \int_{0}^{\pi}\left(\exp (\sqrt{\varepsilon} a \cos \theta) / \lambda_{1}\right)(\sin \theta)^{k-2} d \theta}{\lambda_{1}^{-1} \varepsilon^{(k-3) / 4} \exp \left(-(\sqrt{\varepsilon}-a)^{2} / 2 \lambda_{1}\right)} .
\end{aligned}
$$

And, by Laplace's method [2]

$$
\int_{0}^{\pi} \exp \frac{\sqrt{\varepsilon} a \cos \theta}{\lambda_{1}}(\sin \theta)^{k-2} d \theta \sim \frac{1}{2} \Gamma\left(\frac{k-1}{2}\right)\left(\frac{2 \lambda_{1}}{a \sqrt{\varepsilon}}\right)^{(k-1) / 2} \exp \frac{\sqrt{\varepsilon} a}{\lambda_{1}} .
$$

Hence (4) is

$$
\frac{1}{2} \lambda_{1} \Gamma\left(\frac{k-1}{2}\right)\left(\frac{2 \lambda_{1}}{a}\right)^{(k-1) / 2},
$$

and, consequently, (3) is asymptotic to

$$
\sqrt{\frac{\lambda_{1}}{2 \pi}} a^{(1-k) / 2} \varepsilon^{(k-3) / 4} \exp -\frac{1}{2 \lambda_{1}}(\sqrt{\varepsilon}-a)^{2} .
$$

(The special cases $k=1$ and $k=2$ easily lead to the same expression.)

To sum up, we have

THEOREM 2. If $a>0$, then

$$
\limsup _{\varepsilon \rightarrow \infty} P\left\{\|x-b\|^{2}>\varepsilon\right\} \varepsilon^{1-k / 2} \exp \frac{1}{2 \lambda_{1}}(\sqrt{\varepsilon}-a)^{2}<K_{1},
$$

where $K_{1}$ is defined by (2);

$$
\liminf _{\varepsilon \rightarrow \infty} P\left\{\|x-b\|^{2}>\varepsilon\right\} \varepsilon^{(3-k) / 4} \exp \frac{1}{2 \lambda_{1}}(\sqrt{\varepsilon}-a)^{2} \geqslant \sqrt{\frac{\lambda_{1}}{2 \pi}} a^{(1-k) / 2} .
$$


REMARKS. 1. Zolotarev discussed the limiting behavior of $P\left\{\Sigma_{1}^{\infty} x_{i}^{2}>\varepsilon\right\}$ as $\varepsilon \rightarrow \infty$ in [4], where $\left\{x_{i}\right\}$ are independent $\mathscr{U}\left(0, \sigma_{i}^{2}\right)$ with $\Sigma_{1}^{\infty} \sigma_{i}^{2}<\infty$. This is a special case of Theorem 1 . The proof here is much simpler.

2. It is tempting to try to apply Theorem 3 of [4], but this would require establishing that $e^{\varepsilon /\left(2 \lambda_{1}\right)}(1-F(\varepsilon))$ is nondecreasing, and would still give a weakened version of Theorem 2 . Of course, our theorem also leaves open the question of the exact asymptotic behavior of $1-F(\varepsilon)$, when $a>0$.

Acknowledgement. The Tauberian argument was suggested by Professor Ulf Grenanader.

\section{BIBLIOGRAPHY}

1. W. Feller, An introduction to probability theory and its applications, vol. 2 (2nd ed.), Wiley, New York, 1971.

2. A. Erdélyi, Asymptotic expansions, Dover, New York, 1956.

3. V. M. Zolotarev, Concerning a certain probability problem, Theor. Probability Appl. 6 (1961), 201-204.

4. E. E. Kohlbecker, Weak asymptotic properties of partitions, Trans. Amer. Math. Soc. 88 (1958), 346-365.

Division of Applied Mathematics, Brown University, Providence, Rhode Island 02912

Current address: Institute of Mathematics, Academia Sinica, Taipai, Taiwan, Republic of China 